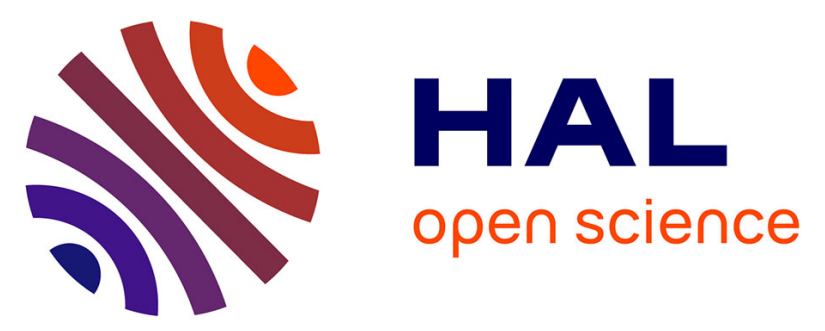

\title{
Autonomous vehicle high-accuracy position and integrity engine performance results
}

\author{
E. Domínguez Tijero, A. Chamorro Moreno, M. T. Fernández Calzón, J. \\ García, J. Ibañez-Guzmán, E. Stawiarski, Ph. Xu, G. Avellone, F. Pisoni, E. \\ Falletti, et al.
}

\section{To cite this version:}

E. Domínguez Tijero, A. Chamorro Moreno, M. T. Fernández Calzón, J. García, J. Ibañez-Guzmán, et al.. Autonomous vehicle high-accuracy position and integrity engine performance results. 32nd International Technical Meeting of the Satellite Division of The Institute of Navigation (ION GNSS+ 2019), Sep 2019, Miami, United States. pp.1234-1241, 10.33012/2019.16872 . hal-02429623

\section{HAL Id: hal-02429623 https://hal.science/hal-02429623}

Submitted on 30 Aug 2021

HAL is a multi-disciplinary open access archive for the deposit and dissemination of scientific research documents, whether they are published or not. The documents may come from teaching and research institutions in France or abroad, or from public or private research centers.
L'archive ouverte pluridisciplinaire HAL, est destinée au dépôt et à la diffusion de documents scientifiques de niveau recherche, publiés ou non, émanant des établissements d'enseignement et de recherche français ou étrangers, des laboratoires publics ou privés. 


\title{
Autonomous Vehicle High-Accuracy Position and Integrity Engine Performance Results
}

\author{
E. Domínguez Tijero, A. Chamorro Moreno, M. T. Fernández Calzón, GMV, Spain; \\ J. García, FICOSA, Spain; \\ J. Ibañez-Guzmán, E. Stawiarski, RENAULT, France; \\ Philippe Xu, University of Technology of Compiègne, France; \\ G. Avellone, F. Pisoni, STMicroelectronics, Italy; \\ E. Falletti, LINKS Foundation, Italy; \\ M. Ortiz, IFSTTAR, France
}

\section{BIOGRAPHY}

Enrique Domínguez Tijero received a M.Sc. degree in Telecommunications Engineering in 2000 and a Master in Space Technologies in 2009, both from the Polytechnic University of Madrid. He joined GMV in 2000 working first in the development of EGNOS and Galileo and since 2009 in GNSS software receivers, multi-sensor fusion algorithms, integrity algorithms and $5 \mathrm{G}$ positioning.

Adrián Chamorro Moreno holds a M.Sc. in Space Systems Engineering from the Technical University of Madrid. He joined GMV in 2017 working in R\&D for ODTS and PPP since then. He joined ESCAPE project and is responsible of the PPP integration in this project.

María Teresa Fernández Calzón holds a degree in Telecommunications Engineering in 2012 from the University of Valladolid. In the same year she joined GMV in the automotive division, working first as software developer in the macro of connected car and including functionalities as Pay as You Drive, Electrical Vehicle management or eCall, and since 2018 as Project Manager also in automotive field.

Jessica García obtained her degree in Telecommunication Engineering from the Universitat Politècnica de Catalunya in 2010. She started as a firmware engineer working on security systems and developed her career on different sectors. In 2014 she got in the automotive sector, where she currently works from the research area of the international company FICOSA.

Javier Ibañez-Guzmán obtained his $\mathrm{Ph} . \mathrm{D}$. at the University of Reading and his MSEE at the University of Pennsylvania (USA) as a Fulbright scholar. In 2011, he was visiting scholar at the University of California, Berkeley (CITRIS). He is member of the technical staff at Renault S.A., working on autonomous vehicle navigation technologies. Formerly he was senior scientist at a national research institute in Singapore, where he spearheaded work on autonomous ground vehicles. Dr. Ibañez-Guzmán has several publications and patents in the robotics and automotive domains.
Emmanuel Stawiarski obtained a degree in Signal Processing from the École Supérieure d'Électricité in France in 1999. He joined Renault in 2003, working first in the electronical development of instrument clusters, then navigation systems. He is currently working in Research department on localisation systems for autonomous driving vehicle projects.

Philippe Xu graduated from the Ecole Normale Supérieure de Cachan, France, in 2011. He received his Ph.D. degree from the Université de Technologie de Compiègne, Heudiasyc UMR CNRS 7253, France, in 2014 and is an associate professor in this same university since 2015. His research interests cover information fusion, computer vision and machine learning applied to autonomous vehicles.

Giuseppe Avellone (Master in Electronics, Università di Palermo, 1996) is with the Functional Safety Management Office of Automotive Product Group at STMicroelectronics. He was previously involved in development of RF IC's, algorithms and applications mainly for GNSS receivers and CDMA/UMTS receivers. His research interests include Functional Safety and signal processing for GNSS receivers and wireless communication systems.

Fabio Pisoni (Master in electronics, Politecnico di Milano, 1994) is leading the GNSS System and Software Team at STMicroelectronics. He was previously with the GNSS System Team in Nemerix SA and has earlier experience in communications. His research interests include DSP and navigation algorithms for embedded GNSS receivers, observables generation and integrity, functional safety.

Emanuela Falletti (Ph. D. in electrical and communications engineering, 2004) is the head of the GNSS Core Algorithms unit in the Navigation Technologies research area of LINKS Foundation, Torino, Italy. Her research activity focuses on digital signal processing algorithms for advanced GNSS software receivers, interference detection and mitigation, multiantenna processing, and signal simulation. 
Miguel Ortiz works as a geolocation research engineer at the COSYS department in the Institut Français des Sciences et Technologies des Transports, de l'Aménagement et des Réseaux (IFSTTAR).

\begin{abstract}
One of the critical functions in autonomous driving is vehicle localisation because, when it is accurate and reliable, it allows the safe navigation of the vehicle when operating without human supervision (SAE Levels 4 and 5 of autonomous driving).
\end{abstract}

This paper presents the performance results obtained when testing in real conditions an innovative positioning engine based on a safety-oriented paradigm. The tested engine employs a mass market dual-frequency GNSS receiver, intelligent off-the shelf automotive cameras, lane-level navigation maps, low-cost inertial sensors and vehicle odometry. A unique feature is the provided integrity layer, estimated in real-time and consisting of Protection Levels (PL), which bound the error of each estimated value with a certain confidence level. This integrity layer is used as part of autonomous vehicle architecture to ensure that the vehicle navigates safely.

The results show the position performances along with the real confidence levels achieved by the estimated Protection Levels. The solution estimated by the engine in real time is based on the dual-frequency measurements obtained from a mass-market GNSS receiver, the measurements of the lateral distance to the lane-markings provided by an intelligent off-the shelf automotive camera, lane-level accurate navigation maps, the measurements obtained from low-cost inertial sensors and the vehicle odometry. With respect to the engine, it is able to process all these measurements by means of:

- A real time PPP hybrid algorithm that employs dualfrequency GPS and Galileo measurements, inertial sensors and PPP corrections obtained from a web server when the connection is available through the cellular network.

- A robust hybrid GNSS standard positioning algorithm allowing to perform consistency checks in parallel

- An algorithm that improves the accuracy by means of the vehicle sensors, the lateral distance measurements to the road lane markings provided by the automotive intelligent camera and the lane-level accurate maps. It is also able to provide an accurate position relative to the map.

The position and orientation values provided by the engine (including the position computed relative to the map) are complemented with the estimation of the associated integrity Protection Levels (PL), computed for multiple target integrity risks. The implementation of an integrity layer is crucial since in safety-critical applications it can be more important to know whether the information is reliable than the precise information itself. This integrity layer determines the degree of usability of the location and orientation estimations, which is used as part of autonomous vehicle architecture to ensure that the vehicle operates safely.

The position and integrity engine has been integrated in an autonomous car and has been tested in real conditions under different environments assessing its performances based on the accurate reference provided by other equipment installed in the car for testing purposes. The paper presents the position and protection level performance results obtained when testing the vehicle under open-sky, sub-urban and deep urban conditions.

The engine employed to obtain the results presented in this paper has been developed and tested with an autonomous car within the ESCAPE project ([1]). ESCAPE (European Safety Critical Applications Positioning Engine) is a project co-funded under the Fundamental Elements program of the European GNSS Agency (GSA). It started on October 2016 with a duration of 3 years and with the main objective of developing a localisation system that provides the vehicle position and attitude estimations to be employed in safety critical applications like Autonomous Driving (AD) or Advanced Driving Assistance Systems (ADAS). The project is led by the Spanish company FICOSA in collaboration with partners from across Europe: Renault, IFSSTAR and the University of Technology of Compiègne (UTC) from France, STMicroelectronics and LINKS Foundation from Italy and $G M V$ from Spain.

ESCAPE enables a high-grade of data fusion (GNSS, inertial sensors, cameras and vehicle sensors) and the exploitation of several key technological differentiators such as the precise point positioning service (PPP), the potential use of the Galileo signal authentication and the provision of an integrity layer to assess the degree of trust one can associate to the position information provided by the device. Therefore, the three key pillars of the safetyoriented navigation technology provided by ESCAPE engine are:

- The smart exploitation of different localization data sources to provide a highly accurate navigation solution, including GPS and Galileo dual-frequency measurements, intelligent cameras providing lateral distance to road lane-marks, inertial measurement units, vehicle odometry, PPP corrections and high definition maps;

- The unique provision of real-time integrity protection levels associated to the location estimates, which express the "degree of usability" of the positioning information for safety-critical applications. The PLs associated to the positions computed relative to the map are fundamental for autonomous driving applications; 
- The full integration of the ESCAPE engine into a vehicle with autonomous driving capabilities, and its test on several different reference paths and environmental conditions.

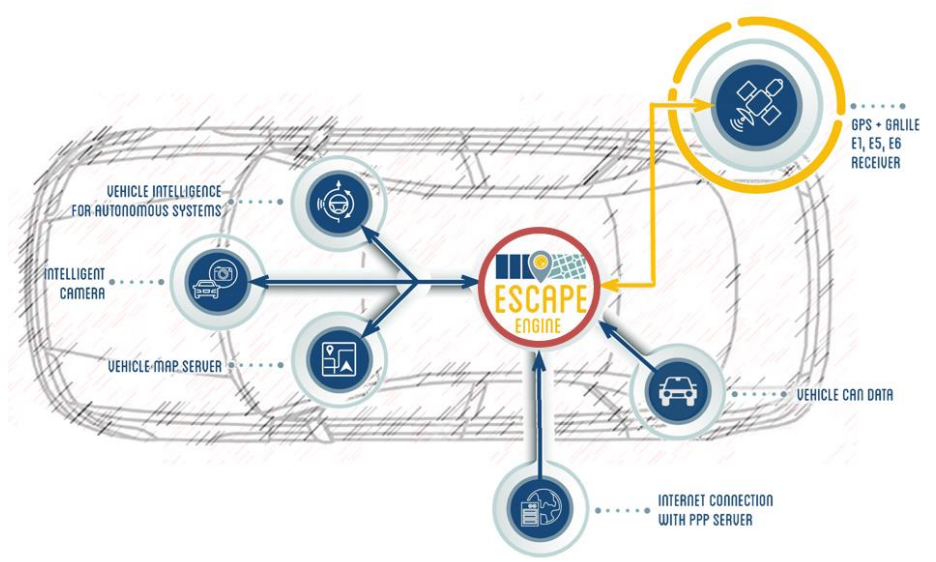

Figure 1 - Conceptual block diagram of the in-car system including the ESCAPE GNSS Engine

The innovative ESCAPE GNSS Engine (EGE) is close-tomarket, its components are organized in a modular architecture and has safety at its core as its specification and design are based on the ISO 26262 recommendations ([2]).

ESCAPE was already introduced in [3]. The following sections provide a brief description of the ESCAPE objectives, its design (system, HW and SW) and the positioning and integrity algorithms (see [3] for further details) before describing the tests that have been carried out and the obtained performance results.

\section{ESCAPE OBJECTIVES}

Main objectives of ESCAPE project [1]:

- Development of a positioning engine for safety critical applications

- Exploration of European GNSS differentiators

- Autonomous driving as the target application

- Provision of integrity as the most relevant performance feature

ESCAPE innovative aspects (see Figure 2) contribute to the achievement of the project objectives.

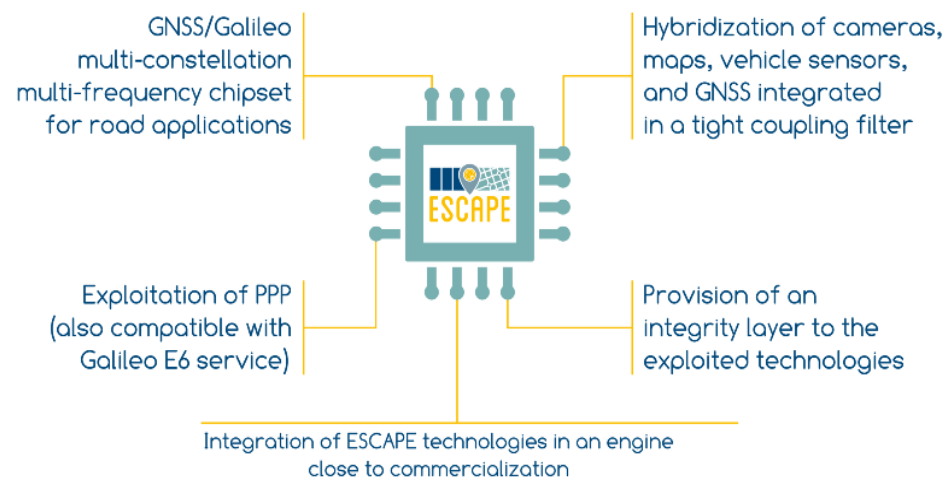

Figure 2 - ESCAPE Innovative Aspects

ESCAPE provides a hybrid solution with an integrity layer making the most of all the sensors that should be in Level $4 \mathrm{SAE}$ vehicles.

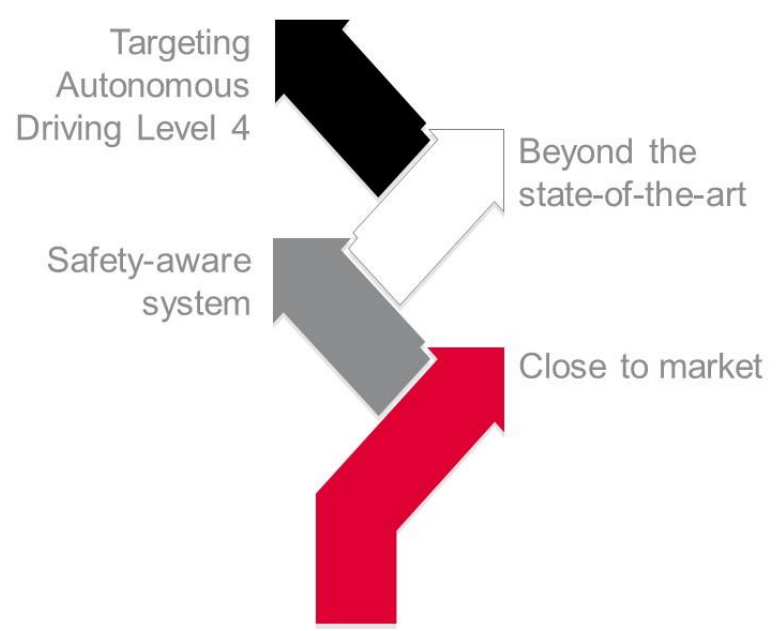

Figure 3 - ESCAPE steps to SAE Level 4

\section{SYSTEM DESIGN}

The design of the ESCAPE GNSS Engine (EGE) is based on three key pillars:

- Use of different data sources:

$\begin{array}{ll}\circ & \text { GNSS } \\ \circ & \text { IMU } \\ \circ & \text { Vehicle Sensors } \\ \circ & \text { Camera (lane markings) }\end{array}$

- Provision of real-time integrity level

- Integration into vehicle 
Three key pillars

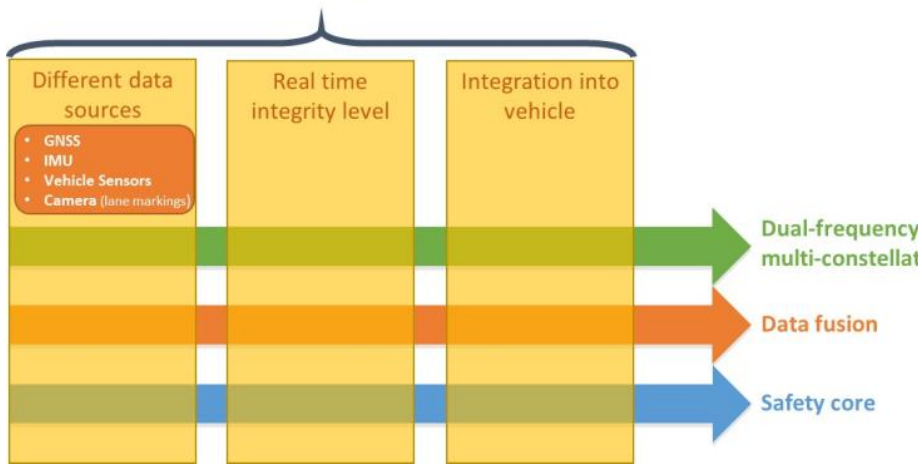

Figure 4 - ESCAPE System Engine


Figure 6 - EGE HW

The whole architecture of the EGE hardware follows the most recent practices in the design of automotive electronic control units, so that all the interfaces, configurations and form factors result compliant with widely recognized sector trends. The safety approach followed in the project during the phases of specification and design is based on the recommendations put in ISO 26262 [2].

\section{HW DESIGN}

ESCAPE GNSS Engine (EGE) hardware includes the following main components:

- The GNSS receiver chipset,

- The Inertial Measurements Unit (IMU)

- The main processor,

- The peripherals needed for the communications of the board with the vehicle and with the cellular network.

EGE components are organized in a modular architecture, in which a main board provides access to the features supported by the EGE, while a core module implements the core processing capabilities. By combining all these components and the navigation and integrity algorithms, the positioning engine is close-to-market and has safety at its core.

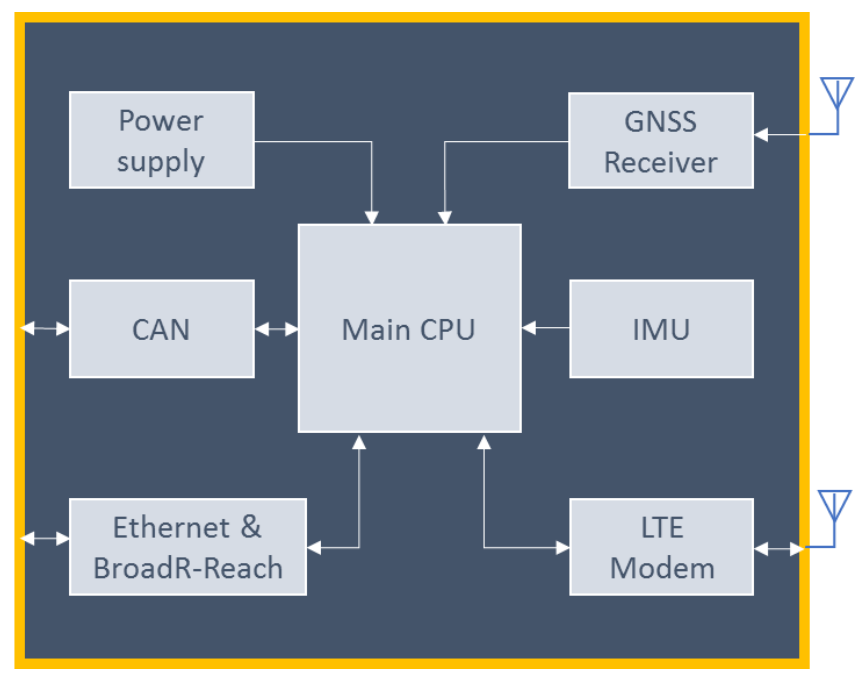

Figure 5 - EGE HW Architecture
ESCAPE GNSS receiver main distinguishing feature is that it is an automotive-grade receiver capable of processing at the same time signals from two different GNSS bands and from different satellite constellations. This capability is common in high-end professional receivers [4] but it represents a cutting-edge industrial development in the automotive Tier- 2 panorama, where it conjugates highly demanding safety requirements to highvolumes and comparatively limited costs and sizes.

ESCAPE GNSS receiver integrates in a unique device a high-end GNSS technology traditionally reserved for professional applications, with the dual-band Galileo processing, as well as all the hardware and software safety aspects that are needed to certify the component for the automotive market [2]. The multi-band GNSS receiver uses a two-chip approach with the Teseo APP-ME (STA9100MGA and STA5635S). See ESCAPE GNSS receiver description in [3] for further details.

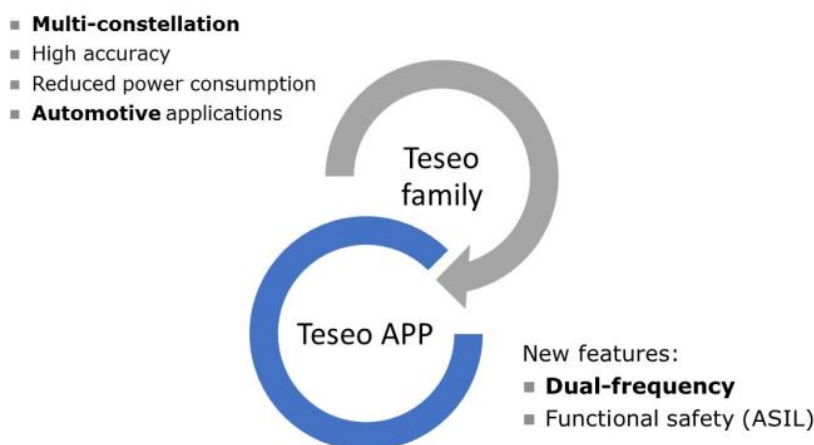

Figure 7 - EGE GNSS Receiver

\section{SW DESIGN}

The EGE software is hosted in a multi-processor SystemOn-Chip, chosen to guarantee the availability of the necessary computational resources at a competitive cost

\section{GNSS RECEIVER}


and its architecture is organized in three layers: the board support package, the middleware layer and the algorithm facility application layer. See ESCAPE SW design description in [3] for further details.

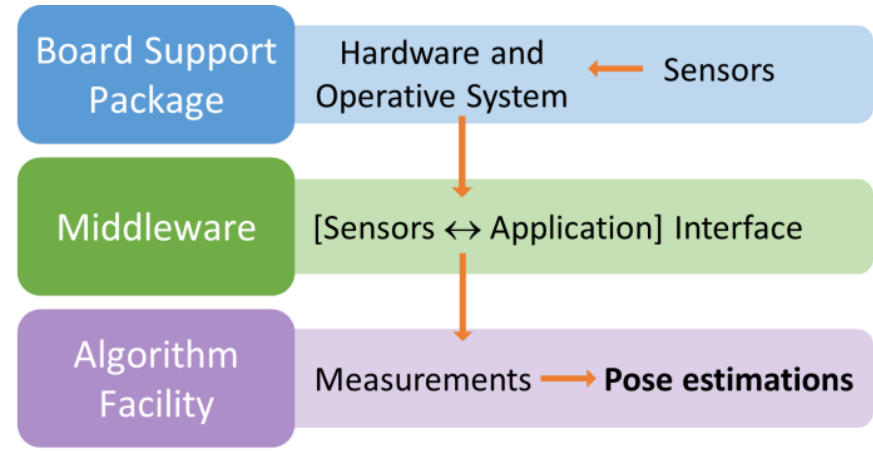

Figure 8 - EGE SW Elements

\section{EGE POSITION\&INTEGRITY ALGORITHMS}

These are the two main position and integrity algorithms employed in the engine:

- The GNSS+Sensors Positioning\&Integrity algorithm, where GNSS measurements are integrated with those provided by an inertial measurement unit (IMU) in order to provide a GNSS pose with integrity. This position is computed by combining Standard Point Positioning (SPP) and Precise Point Positioning (PPP) algorithms. The PPP algorithm exploits an Internet connection to a remote server to get sets of precise corrections for the GNSS navigation: when available, the PPP service is able to reach decimeter-level positioning accuracy [5]. The PPP algorithm is based on the real-time magicPPP ([6]).

- The Camera-based Positioning\&Integrity algorithm, enabled by the availability of accurate maps containing road lane-marks. These maps along with the intelligent camera measurements, which provide the lateral distance to the lane-marks, allow a second positioning service with accuracies that can reach a few centimeters in the cross direction. The algorithm also employs speed and yaw rate measurements from vehicle sensors to reach the maximum possible accuracy. Thus, the computed solution complements and enhances the positions provided by the GNSS+Sensors Positioning\&Integrity algorithm.

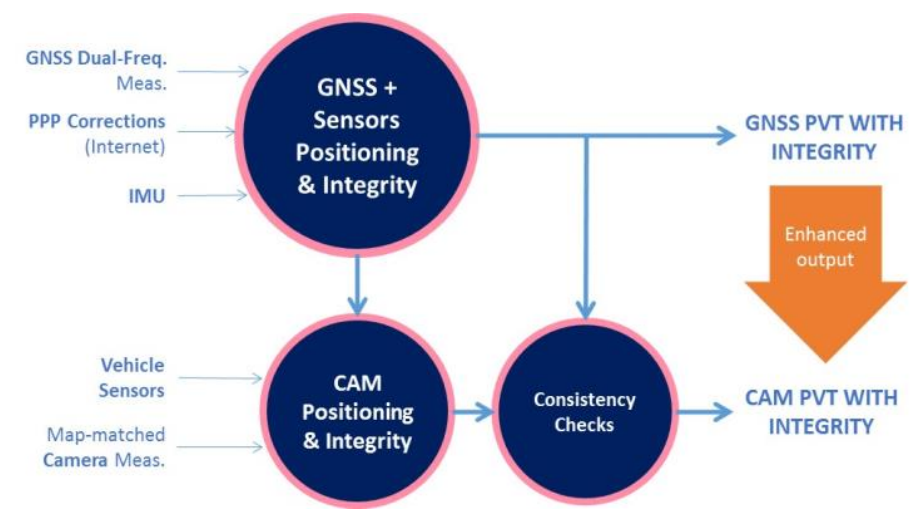

Figure 9 - EGE Position\&Integrity Algorithms Architecture

\section{Integrity Layer: Protection Levels}

The integrity of a certain estimate refers to the confidence one can give to the correctness of the estimate with respect to the true (but unknown) quantity. This confidence is expressed in the language of GNSS positioning with the concepts of protection level (PL) and integrity risk (IR). The PL should bound the error with a certain confidence level and the IR is the probability that the error exceeds the protection level [7]. A well-established and trustable framework to set integrity risks and compute protection levels is mandatory for any applications wants to use an estimated position as an input for operations involving safety of life (as well as economical transactions or any kind of law enforcement, though with different levels of risk).
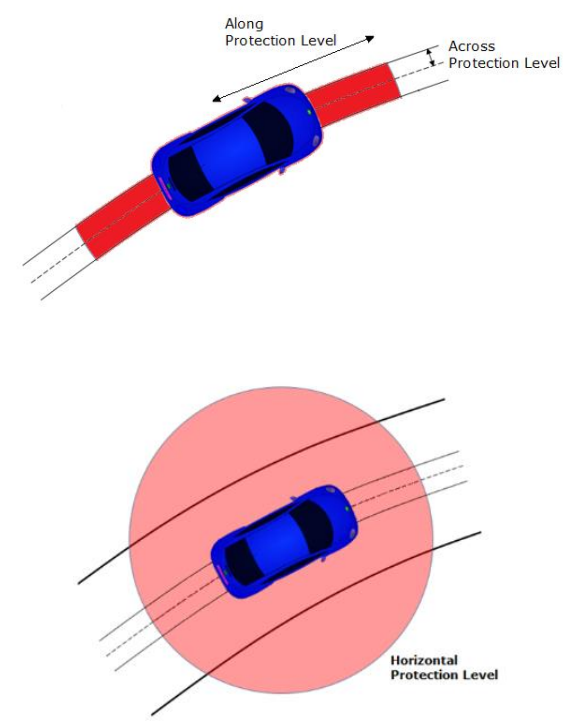

Figure 10 - Protection Level concept associated to an estimated position

For this reason, the EGE provides each position estimate, whatever service is used to compute it, with a protection level. The protection levels, provided by the "GNSS+Sensors Positioning\&Integrity" and the "CAM 
Positioning\&Integrity" algorithms, are computed with a technique known as KIPL (Kalman Integrated Protection Level), based on the idea of dynamically modelling the different components of the positioning error with a properly parameterized error probability distribution ([8], [9] and [10]). Each distribution is processed and updated separately and provides a contribution to the total error probability. Finally, the protection level is computed as the maximum error level whose probability is below a given integrity risk. Thus, the integrity module forwards the integrity solution in form of a set of protection levels corresponding to different target integrity risks (TIRs).

One way to use these protection levels is to raise an alert when the PL corresponding to a certain integrity risk becomes greater that the alarm limit required by the user depending on the circumstances.

\section{ESCAPE TESTS}

The developed EGE was integrated in the UTC-Renault autonomous car and the tests took place in July 2019 at the University of Technology of Compiègne (UTC). The autonomous car employed in the tests also had a high-grade trajectory reference system (GNSS+high-grade IMU) which post-processed $\mathrm{cm}$-error solution has been employed as the truth reference trajectory when assessing EGE performances.

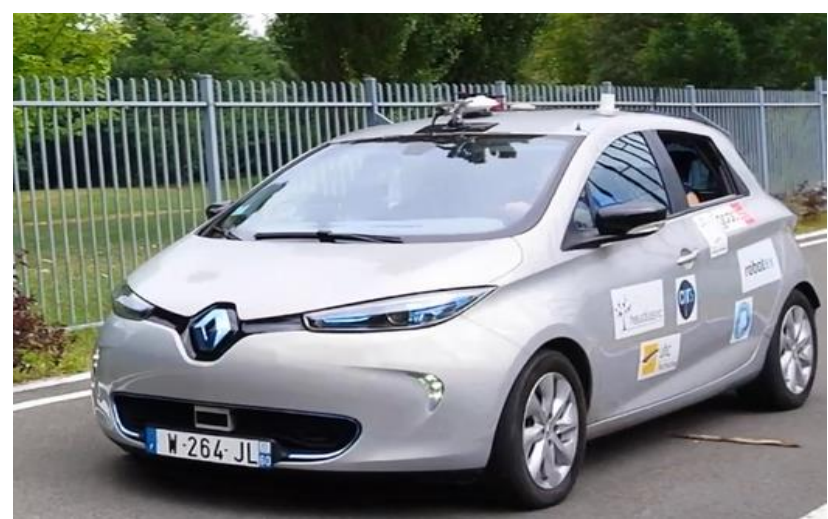

Figure 11 - Autonomous Car employed in ESCAPE tests

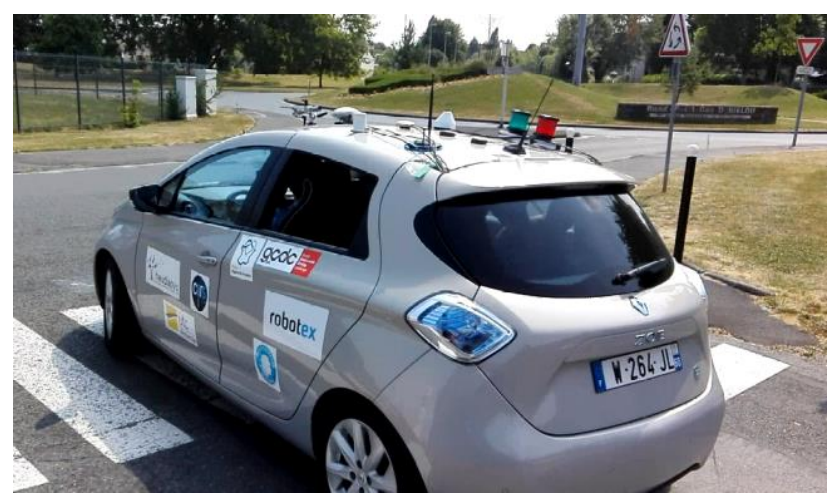

Figure 12 - ESCAPE Tests at the University of Technology of Compiègne
The tests were performed following a route in Compiègne covering three different environments:

- Open-Sky

- Sub-Urban

- Urban

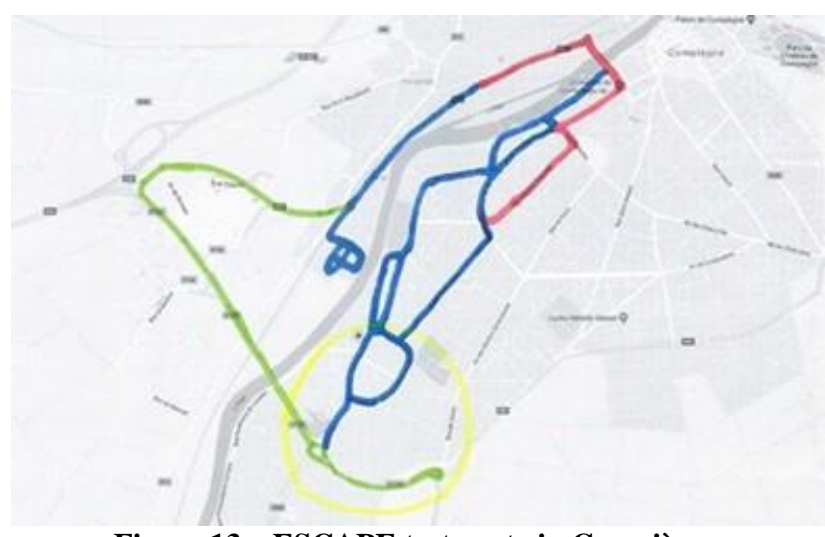

Figure 13 - ESCAPE test route in Compiègne

When assessing the obtained results it shall be taken into account that the EGE was tested with the GNSS receiver tracking L1/E1 and L5/E5a bands so only a maximum of 7-9 dual-frequency (DF) SVs were in view:

- 3-5 GPS L1\&L5 SVs

- 3-5 Galileo SVs

Final EGE GNSS receiver will be capable of tracking GPS L1\&L2C and Galileo E1\&E5b, thus increasing the number of available dual-frequency GPS SVs.

Besides, it also shall be taken into account that camera measurements (lane-marks) were usually available in open-sky roads but not available in roundabouts and nonuniformly available in sub-urban and urban streets.

The following figures and tables provide the results obtained with the EGE in each environment running the PPP and enhancing the computed solution with the camera measurements and the vehicle sensors.

Open-Sky
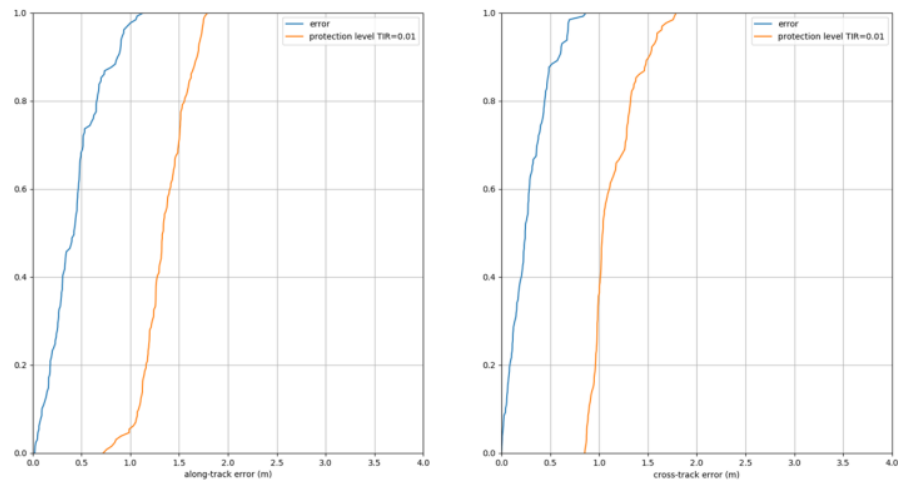

Figure 14 - Open-Sky along/longitudinal and cross/lateral error and PL CDF 

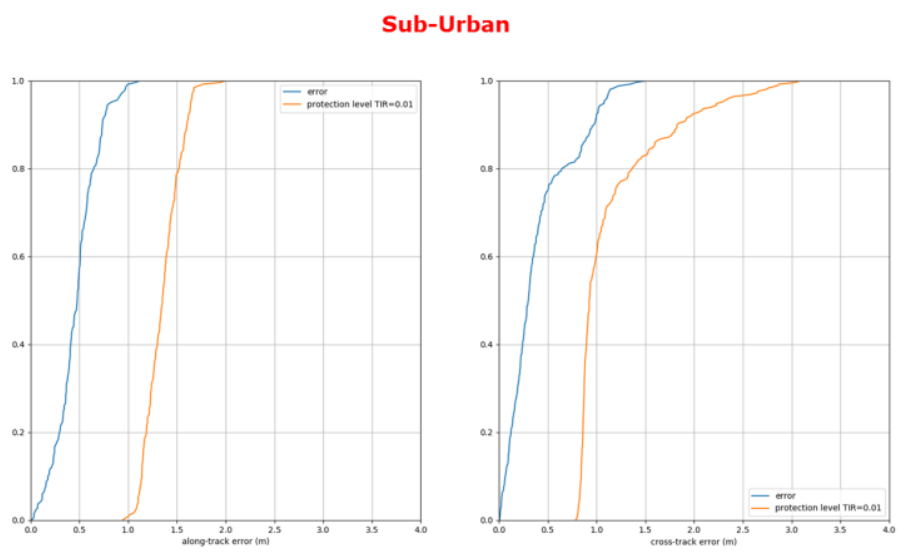

Figure 15 - Sub-Urban along/longitudinal and cross/lateral error and PL CDF

Urban
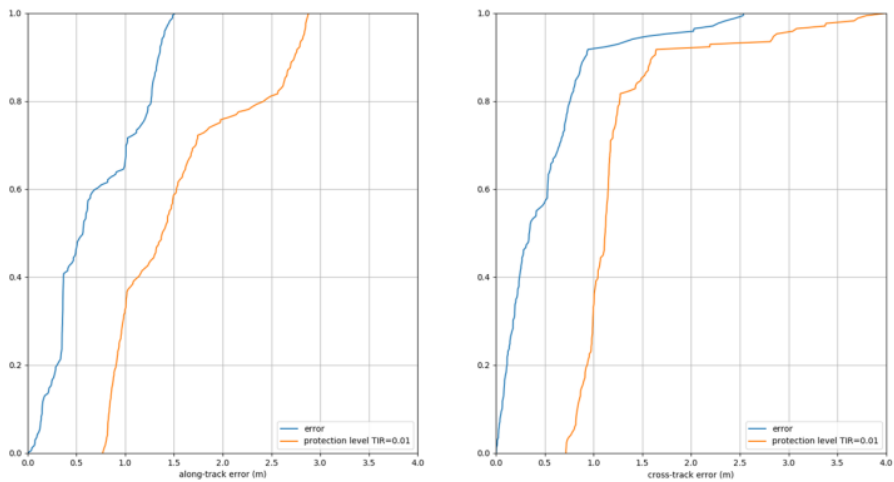

Figure 16 - Urban along/longitudinal and cross/lateral error and PL CDF

The results show how, in open-sky conditions, the along and cross errors are below $1 \mathrm{~m}$, which is a very good result taking into account there are only 6-9 dual-frequency SVs in view. Worst results were obtained while driving around the roundabouts where no camera measurements were available. In sub-urban, the lower amount of camera measurements increases the cross/lateral errors, while in urban, besides having fewer camera measurements, the limited number of satellites in view due to the buildings increases the error in both dimensions. Nevertheless, suburban errors are close to $1 \mathrm{~m}$ and almost all urban errors are below $2 \mathrm{~m}$, which are excellent results.

\begin{tabular}{|c|c|c|c|c|c|c|}
\hline \multicolumn{7}{|c|}{$\begin{array}{c}\text { PPP + Vehicle Sensors + Camera } \\
\text { (6-9 GNSS DF SVs in view) }\end{array}$} \\
\hline \multirow{2}{*}{ Percentile } & \multicolumn{3}{|c|}{$\begin{array}{c}\text { Along/Longitudinal Error } \\
(\mathbf{m})\end{array}$} & \multicolumn{3}{c|}{ Cross/Lateral Error } \\
\cline { 2 - 7 } & \multicolumn{3}{|c|}{ (m) } \\
\cline { 2 - 8 } & Open-Sky & Sub-Urban & Urban & Open-Sky & Sub-Urban & Urban \\
\hline $50 \%$ & 0.42 & 0.47 & 0.56 & 0.24 & 0.30 & 0.34 \\
\hline $75 \%$ & 0.59 & 0.59 & 1.17 & 0.41 & 0.50 & 0.70 \\
\hline $95 \%$ & 0.92 & 0.82 & 1.40 & 0.67 & 1.07 & 1.65 \\
\hline
\end{tabular}

Table 1 - EGE Performance Test Results along/longitudinal and cross/lateral errors

\begin{tabular}{|c|c|c|c|c|c|c|}
\hline \multicolumn{7}{|c|}{$\begin{array}{l}\text { PPP + Vehicle Sensors + Camera } \\
\text { (6-9 GNSS DF SVs in view) }\end{array}$} \\
\hline \multirow{2}{*}{ Percentile } & \multicolumn{3}{|c|}{ Along/Longitudinal PL (m) } & \multicolumn{3}{|c|}{ Cross/Lateral PL (m) } \\
\hline & Open-Sky & Sub-Urban & Urban & Open-Sky & Sub-Urban & Urban \\
\hline $50 \%$ & 1.33 & 1.35 & 1.38 & 1.03 & 0.92 & 1.12 \\
\hline $75 \%$ & 1.51 & 1.48 & 1.96 & 1.29 & 1.19 & 1.22 \\
\hline $95 \%$ & 1.72 & 1.64 & 2.83 & 1.59 & 2.26 & 2.86 \\
\hline
\end{tabular}

Table 2 - EGE Performance Test Results along/longitudinal and cross/lateral PLs

The size of the computed protection levels is also very low and they satisfy the integrity risk for which they were computed. Figure 17 and Figure 18 show how the Protection Levels (PL) computed in real-time by the EGE algorithms successfully bound the error of the estimated positions.

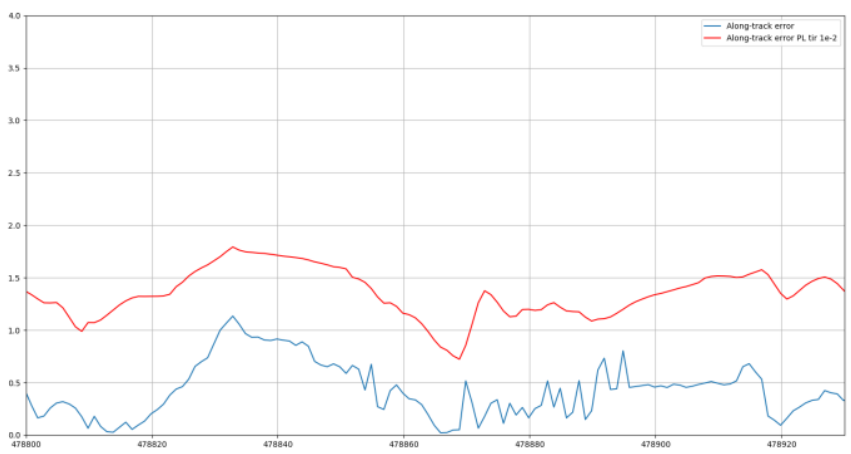

Figure 17 - Example showing how along/longitudinal errors are bounded by the computed PLs

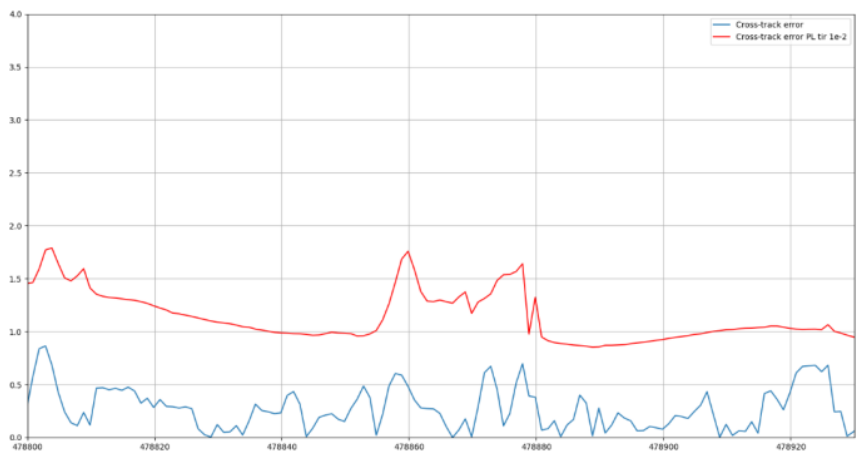

Figure 18 - Example showing how the cross/lateral errors are bounded by the computed PLs

\section{CONCLUSIONS}

The positioning engine developed in the ESCAPE project is capable of providing a hybrid solution with an integrity layer making the most of all the sensors that should be present in Level $4 \mathrm{SAE}$ vehicles. Hence, this innovative 
positioning engine enables automated driving functions up to a SAE automation level 4 and creates a new paradigm of safety-oriented navigation technology on the road.

Safety is the crucial aspect of automated vehicles and ESCAPE engine has developed a state-of-the-art safetyoriented navigation technology based on:

- The smart exploitation of different localization data sources to provide a highly accurate navigation solution, including GPS and Galileo dual-frequency measurements, intelligent cameras providing lateral distance to road lane-marks, inertial measurement units, vehicle odometry, PPP corrections and high definition maps;

- The unique provision of real-time integrity protection levels associated to the location estimates, which express the "degree of usability" of the positioning information for safety-critical applications. The PLs associated to the positions computed relative to the map are fundamental for autonomous driving applications;

- The full integration of the ESCAPE engine into a vehicle with autonomous driving capabilities, and its test on several different reference paths and environmental conditions.

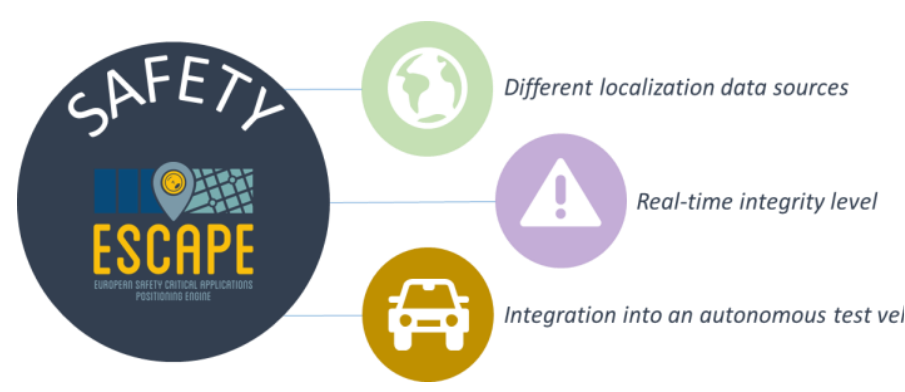

Figure 19 - New paradigm of safety-oriented road navigation technology

In order to give insight of the developed technology and to show the achieved results to the automated vehicle experts and GNSS community, the ESCAPE project will celebrate a demonstration day on the $27^{\text {th }}$ of November 2019 at UTC Compiègne, details will be published in [1].

\section{ACKNOWLEDGMENTS}

The European Safety Critical Applications Positioning Engine (ESCAPE) is funded by the European GNSS Agency (GSA) under the European Union's Fundamental Elements research and development programme.

\section{REFERENCES}

[1] ESCAPE (European Safety Critical Applications Positioning Engine) Project Website:

http://www.gnss-escape.eu/

[2] International Standard Organization (2011) Road vehicles -- Functional safety. ISO 26262.

[3] Domínguez Tijero, E. et al., "High Accuracy Positioning Engine with an Integrity Layer for Safety Autonomous Vehicles," Proceedings of the 31st International Technical Meeting of the Satellite Division of The Institute of Navigation (ION GNSS+ 2018), Miami, Florida, September 2018, pp. 1566-1572.

[4] Gleason, S., and Gebre-Egziabher, D. (2009), GNSS Applications and Methods. Norwood, MA: Artech House.

[5] Domínguez Tijero, E. et al., "Advanced GNSS Algorithms for Safe Autonomous Vehicles". Proceedings of the 30th International Technical Meeting of The Satellite Division of the Institute of Navigation (ION GNSS+ 2017), Portland, Oregon, September 2017, pp. 655-664.

[6] magicPPP webpage:

https://www.gmv.com/en/Products/magicPPP/

[7] European GNSS Agency (2015) Report on the performance and level of integrity for safety and liability critical multi-applications. May 2015.

[8] Method for computing an error bound of a Kalman filter based GNSS position solution, Pending Patent: Europe EP14189240.6.

[9] Navarro Madrid, P. F., et al., "Computing Meaningful Integrity Bounds of a Low-cost Kalman-filtered Navigation Solution in Urban Environments". Proceedings of the 28th International Technical Meeting of The Satellite Division of the Institute of Navigation (ION GNSS+ 2015), Tampa, Florida, September 2015, pp. 2914-2925.

[10] Navarro Madrid P. F. et al., "PPP Integrity for Advanced Applications, Including Field Trials with Galileo, Geodetic and Low-Cost Receivers, and a Preliminary Safety Analysis". Proceedings of the 29th International Technical Meeting of The Satellite Division of the Institute of Navigation (ION GNSS+ 2016), Portland, Oregon, September 2016, pp. 3332-3354. 